\title{
Germanica
}

\section{Georg Trakl : les poèmes du non-retour}

Georg Trakl: die Gedichte der Heimkehr

Jean-Daniel Krebs

\section{(2) OpenEdition}

Journals

Édition électronique

URL : http://journals.openedition.org/germanica/2803

DOI : 10.4000/germanica.2803

ISSN : 2107-0784

\section{Éditeur}

Université de Lille

\section{Édition imprimée}

Date de publication : 30 juin 1987

Pagination : 35-48

ISSN : 0984-2632

\section{Référence électronique}

Jean-Daniel Krebs, « Georg Trakl : les poèmes du non-retour », Germanica [En ligne], 1 | 1987, mis en ligne le 16 octobre 2015, consulté le 06 octobre 2020. URL : http://journals.openedition.org/ germanica/2803 ; DOI : https://doi.org/10.4000/germanica.2803

Ce document a été généré automatiquement le 6 octobre 2020.

(C) Tous droits réservés 


\section{Georg Trakl : les poèmes du non- retour}

Georg Trakl: die Gedichte der Heimkehr

Jean-Daniel Krebs

\section{NOTE DE L'ÉDITEUR}

Les poèmes cités renvoient aux textes établis par Walther Killy et Hans Szkle. NAR, Historisch-kritische Ausgabe, Salzburg, O. Müller, 1969 (2 vol.). Abréviation : H.K.A.

On pourra en consulter la traduction française dans : Georg Trakl, Euvres complètes, trad. de l'allemand par Marc Petit et Jean-Claude Schneider, Paris, Gallimard, 1972.

The night is long that never finds the day

Macbeth, IV, 3.

E.T., la créature fantastique du film de Steven Spielberg, ne nous paraît jamais aussi humaine que lorsque s'exprime son désir de rentrer à la «maison». Le scénariste a prêté à l'extra-terrestre cette image nostalgique de la maison qui, enfouie au plus profond de l'être, oriente selon G. Bachelard les pensées, les souvenirs et les rêves de l'homme :

Et toujours, en nos rêveries, la maison est un grand berceau ${ }^{1}$.

Plusieurs poèmes de Trakl, qui mentionnent le « retour chez soi » («Heimkehr») dans leur titre, s'enracinent dans cette nostalgie primordiale, mais c'est pour circonscrire un vide, une exclusion, l'impossible retour de l'« étranger » qui poursuit son «errance inquiète dans un désert de pierres loin des villages du soir, loin des troupeaux rentrant au bercail " (offenbarung und Untergang). Ces textes ne "traitent" pas le thème du retour : ils relèvent une trace perdue, poursuivant une enquête incertaine sur les voies $\mathrm{du}$ souvenir selon un cheminement qui, au sens premier, est anagogique. L'accueil, le repos, la coïncidence enfin retrouvée de l'être avec ses origines, tout le poids de symbole et d'adhésion profonde porté par la maison, cet élément fondamental d'une poétique de l'espace, s'offre, en revanche, dans un autre poème, Ein Winterabend, 
parabole du miracle accompli, du retour réussi. Cette évocation fabuleuse du terme de l'errance servira d'introduction aux textes du non-retour; sa vision lumineuse permet de mieux mesurer le tragique détour par lequel le retour s'effectue dans la réalité de l'œuvre et de l'existence de Trakl.

Ein Winterabend (2e version)

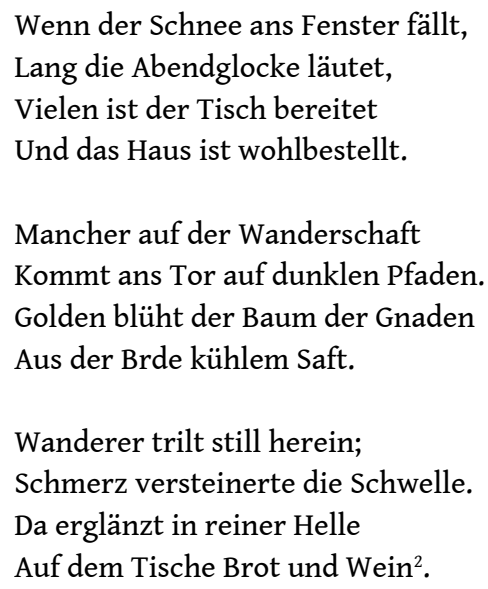

3 Ce conte de Noël - légende émerveillée - montre la grâce en action: l'élection inouïe parmi la foule des pélerins de celui qui, seul, aura le privilège d'entrer, pour qui seul s'accomplit l'absolu retour à la maison; l'histoire de cet espoir insensé, mais exaucé, n'est pas sans rappeler certaines paraboles kafkaïennes de la grâce. Selon un passage progressif du dehors au dedans, signalé par la fenêtre, la porte et le seuil, le choix se précise tandis que s'intensifie le rayonnement du don lumineux: "pour beaucoup » de ceux qui attendent dans la neige la maison est prête (strophe 1), " plus d'un » d'entre ceux qui marchent dans la nuit parviendra à la porte et verra briller l'arbre de grâce (strophe 2), mais il n'est donné qu'à l'« errant », une des figures habituelles désignant l'auteur, de franchir le seuil et d'atteindre le tabernacle (strophe 3). Un vers cependant dresse un obstacle qui, ici emporté par le flot de la grâce, se constituera ailleurs en une barrière : le seuil pétrifié de douleur (vers 10). Le retour se heurte à ce seuil qui n'est franchissable que dans la souffrance, et ce lieu où se mêlent confusément la douleur et l'espoir, à la limite entre les ténèbres du dehors et la lumière du dedans, est celui où se situe l'œuvre poétique de Trakl.
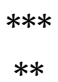

Les textes que leur titre consacre au retour sont structurellement apparentés, car ils présentent un exemple de cette sorte de génération organique qui a été mise en évidence par W. Killy dans l'œuvre de Trakl: divers poèmes apparaissent par ramification à partir du tronc commun d'un ou de plusieurs textes d'origine. Dans le cas présent un groupe de poèmes s'est constitué selon un processus simultané de filiation et de contamination à partir d'un premier texte intitulé Heimkehr. Cette maturation thématique s'étend sur une courte période: entre mai et juin 1914. Pourtant la chronologie des textes, telle qu'elle a pu être reconstituée, révèle une évolution rapide, un phénomène de dégradation souvent relevé dans l'écriture de Trakl, mais en règle générale à l'échelle du poème ou encore pour l'ensemble de l'œuvre. Ici c'est un « cycle » qui, à mesure qu'il se développe, se corrompt comme s'il découvrait en soi un 
principe destructeur. Le texte de Heimkehr recèle en effet, comme un mal endogène, une malédiction attachée au souvenir, qui suscite la poursuite du travail poétique et en même temps le gangrène. Dans ce processus de désagrégation créatrice deux lignées de poèmes se détachent du tronc originel: d'une part les trois versions de Herbstliche Heimkehr, qui évoluent peu de l'une à l'autre, et d'autre part la filiation qui conduit à Die Heimkehr par une ébauche intermédiaire au titre resté comme en suspens - An - (dont certains éléments viennent aussi se greffer sur la branche cousine de Herbstliche Heimkehr) ${ }^{3}$. Aucune de ces pièces n'a été publiée du vivant de Trakl ; seul Die Heimkehr a paru, peu après sa mort, dans le Brenner, la revue d'avant-garde publiée à Innsbruck par L. von Ficker, l'ami et mécène de l'auteur"

5 Heimkehr, Herbstliche Heimkehr et Die Heimkehr constituent donc une trilogie cohérente de poèmes du retour, qui, outre leur parenté génétique, s'ordonnent selon une logique interne. La dégradation signalée inverse en effet le mouvement qui - distribué entre les trois strophes de Ein Winterabend- conduisait des ténèbres à la lumière. Inéluctablement le chemin du retour s'incurve, devient retournement et s'enfonce dans la nuit. Le texte originel, Heimkehr, est encore nimbé de la lumière d'or qui s'offre à celui qui croit avoir franchi le seuil de la paix intérieure. De cette illusion dorée ne subsiste dans Herbstliche Heimkehr qu'un reflet lointain, pareil à celui qui émane de l'«arbre de grâce" aperçu à la fenêtre dans la nuit. Enfin, avec Die Heimkehr, les ténèbres se referment sur l'étranger qui salue en la tombe son ultime espoir. Contrairement à Ein Winterabend où la grâce était offerte, ce groupe de poèmes jalonne la dure démarche de la foi : au don prodigieux s'oppose la recherche exigeante, la poursuite d'une grâce qui se refuse et qu'un espoir irréductible va traquer jusqu'au fond du désespoir. Négligeant les portes qui s'ouvrent sur des demeures de hasard et leur repos illusoire, la quête opiniâtre devient destructrice et conduit par un itinéraire inversé au néant, comme au dernier refuge de l'être, comme au seul retour possible.

6 Le texte intitulé An, qui fait fonction d'une sorte de relais entre Heimkehr et les poèmes dérivés, est particulièrement révélateur par son caractère même d'inachèvement ${ }^{5}$. Il dégage en effet le noyau infrangible autour duquel s'organisent ces poèmes: le souvenir d'où émane l'appel au retour et où se manifeste aussi son impossibilité. Débarrassée de l'or fallacieux du premier poème (Heimkehr), cette ébauche n'est pas encore saturée d'ombre: elle se situe dans un clair-obscur défini d'emblée par l'opposition « douleur et espoir ». Un déroulement chronologique s'y esquisse que l'on retrouve au centre des différents poèmes du retour et qui les désigne comme autant de tentatives de franchir un seuil. Au soleil couchant une habitation, symbolisée par une charpente festonnée de dahlias, garde le souvenir de sombres années; mais, ancré plus anciennement en ces lieux, le visage de l'enfance au doux regard insistant apparait, déclenchant des pleurs qui s'épanchent en une rosée continue ; alors montent de la pénombre de mystérieux appels; enfin, étoile dans la nuit, une lueur apaisante émane du sein même de la mélancolie.

7 Cette déploration des années évanouies au déclin du jour présente encore des traits d'un décadentisme nostalgique appelé à disparaitre face au durcissement ultérieur du thème; de même l'image du soleil se couchant « comme un casque d'or tomberait d'un front ensanglanté » trahit un souci d'esthétisme, dans le goût du Jugendstil, que l'expérience tragique du non-retour va engloutir. La démarche présentée indique cependant clairement que le retour mène en direction de l'enfance, sa "maison", son être perdu et - dans un premier temps - vers l'acceptation mélancolique de cette 
disparition. L'évocation du retour s'enracine ainsi dans un thème général caractéristique de l'œuvre de Trakl, celui du déclin; mais alors que les poèmes du déclin s'abandonnent à la dérive du temps, le retour en remonte le cours au long du fil conducteur du souvenir. C'est une tentative de "rentrer chez soi » en entrant en soi «Einkehr») et, dans l'impossibilité de retrouver la demeure ancienne ruinée, la quête nécessaire d'une autre demeure en des terres devenues inhabitables.

La première tentative de retour, avec Heimkehr, est placée dans une sorte de cadre doré formé par les deux strophes qui enveloppent, et neutralisent, l'aiguillon du souvenir lancinant qui occupe le centre du poème. De l'or en effet émane du soir d'automne apaisant évoqué en entrée et se retrouve, à la fin, transmué en un or intérieur où l'auteur croit avoir trouvé une « demeure».

Heimkehr

Wenn goldne Ruh der Abend odmet

Wald und dunkle Wiese davor

Ein Schauendes ist der Mensch,

Ein Hirt, wohnend in der Herden dämmernder Stille,

Der Geduld der raten Buchen;

So klar da es Herbst geworden. Am Hügel

Lauscht der Einsame dem Flug der Vögel,

Dunkler Bedeutung und die Schatten der Toten

Haben sich ernster um ihn versarmmelt ;

Mit Schauern erfüllt ihn kühler Resedenduft,

Die Hütten der Dörfler der Hollunder,

Wo vor Zeiten das Kind gewohnt.

Erinnerung, begrabene Hoffnung

Bewahrt dies braune Gebälk,

Darüber Georginen hangen,

Dass darnach er die Hände ringe,

Im braunen Gärtchen den schimmernden Schritt

Verboten Lieben, dunkles Jahr,

Dass von blauen Lidern die Tränen stürzten

Dem Fremdling unaufbaltsam.

Von braunen Wipfeln tropft der Tau,

Da jener ein blaues Wild am Hügel erwacht,

Lauschend den lauten Rufen der Fischer

Am Abendweiher

Dem ungestalten Schrei der Fledermäuse;

Aber in goldener Stille

Wohnt das trunkene Herz

Seines erhabenen Todes voll ${ }^{6}$.

Le cadre scellant l'exploration mnémonique s'attarde à camper un paysage tout de quiétude mêlant à ses grandes lignes d'inspiration néo-romantique des éléments de la mythologie personnelle de l'auteur. Mais la sérénité du tableau n'est qu'apparente : la faille se manifeste dans le personnage contemplatif, ce berger qui a établi sa demeure dans la vaste paix de la nature qui l'enveloppe et avec laquelle il se confond pour n'être plus qu' « une chose qui contemple ", un élément de l'univers abîmé dans la communion avec sa propre identité (vers 1-6). Illusion pourtant que cette unité essentielle puisque 
l'homme se mue en "solitaire ", une des désignations par antonomase de l'auteur ; son inquiétude, encore secrète, perce quand il interroge le vol des oiseaux, guetteur cerné par les ombres qui sont autant de souvenirs des années mortes (vers 7-9). Le parfum du réséda qui le surprend alors est un de ces repères poétiques qui chez Trakl signalent un éveil de la conscience : porté ici par un souffle froid, il déclenche l'angoisse (vers 10). Un vertige naît à la vue de ces chaumières et du sureau - un des arbres tutélaires de l'enfance dans cette poésie - entraînés par le temps vers le gouffre du néant. C'est là que demeurait l'enfant et ainsi est emportée sa "maison », le premier, le seul berceau de l'être (vers 11-12).

Alors monte du fond de la mémoire l'image de l'enfance dont l'émergence s'annonçait dans l'ébranlement progressif de la quiétude précédente. Ainsi s'engage le retour qui est d'abord une exploration du souvenir, une exhumation d'" espérances ensevelies». Les années d'enfance sont intimement liées à ces enclos où l'être a trouvé sa forme première: la maison et le jardin. Ce sont ces deux endroits qui en conservent le souvenir selon une syntaxe subtilement ambiguë suggérant qu'inversement aussi la mémoire conserve la charpente brune de la maison (vers 13-14); celle-ci perd ainsi de sa réalité matérielle, ce qui rend vaine toute enquête sur une visite effective de Trakl à sa demeure natale, qui aurait pu être à l'origine de cette réminiscence automnale. Il importe peu que ce pélerinage aux lieux habités par l'enfance repose sur un retour réel au cadre salzbourgeois. L'auteur nous entraîne loin de sa ville natale dans un espace intérieur où maison et jardin sont l'image d'une perfection perdue de l'être, vision fleurie vers laquelle des mains se tendent convulsivement (vers 15-16).

Puis, sans transition, c'est la rupture: le seuil de la maison franchi sans espoir de retour. Le "cheminement lumineux » dans le jardin de l'enfance débouche sur une plongée brutale dans la pénombre selon un raccourci elliptique caractéristique du style de l'auteur : « dans le jardinet brun le pas lumineux amour interdit, sombre année » (vers 17-18). Le rappel d'un " amour interdit» désigne certainement la relation incestueuse du jeune Georg avec sa sœur Grete (une variante plus explicite écrit: «des verboten Liebenden »). Cette expérience décisive, car vécue comme l'irruption du mal, ouvre une blessure jamais refermée. Elle introduit les sombres années, celles d'une adolescence tourmentée, succédant à la période où «l'enfance reposait, paisible, dans une grotte bleue " (Kindheit). C'est l'évocation brutale de ce qui constitue la trame des poèmes du déclin: la corruption de l'innocence (Elis) ou son assassinat (Kaspar Hauser Lied). De même la forme verbale, employée ici encore au passé («stürzten») pour désigner le jaillissement des larmes, renvoie aux racines du mal, au point de non-retour marqué par l'expulsion hors du jardin d'innocence de celui qui désormais se nomme l'« étranger » (vers 19-20).

12 Pourtant les larmes jaillissent de paupières qui ont gardé, ou retrouvé par le souvenir, la couleur bleue, symbole de pureté dans la palette poétique de Trakl: c'est précisément parce qu'ils contiennent un reste d'innocence que ces yeux pleurent l'innocence perdue. Ces larmes suggèrent que le retour est à la fois proche, nécessaire et pourtant impossible : elles ne sont pas la déploration élégiaque d'un passé aboli, mais le désespoir qui jaillit de l'évidence de la grâce aujourd'hui refusée. La demeure de l'enfance n'est pas un simple souvenir effrité par le temps: elle s'impose par la présence lancinante de son absence, par le deuil que suscite sa perte irrémédiable.

13 La rosée qui, dans la dernière partie, s'égoutte des arbres est une image familière du code poétique trakelien : c'est cette même rosée qui - noire - tombe sur les tempes du 
cadavre d'Elis (An den Knaben Elis) et accompagne l'irruption de l'horreur (Der Herbst des Einsamen). Ici encore, quoique traité sur le mode mineur, le thème signale la dissipation d'une illusion: la nature environnante s'est assombrie, la paix qu'elle offrait a été dénoncée comme trompeuse par le retour aux sources pures de l'enfance, avant le saccage du jardin par le mal « Celui-là », qui s'était enfoncé dans un songe au flanc de la colline, se retrouve autre, métamorphosé : animal bleu purifié par le contact onirique avec son être premier (vers 21-22); mais en même temps sa conscience aiguisée s'est ouverte à l'obscure signification précédemment guettée dans le vol des oiseaux et il devine son destin : le déclin l'appelle aussi vers le gouffre par la voix de ces pêcheurs au bord du lac crépusculaire (vers 23-24); plus inquiétant, le cri des chauves-souris se fait l'écho insistant de leurs appels (vers 25). Le retour n'a été qu'un leurre: on ne revient jamais à la maison emportée par l'universelle ruine.

Mais, si la maison de l'enfance est engloutie, si la béatitude d'une nouvelle demeure de stabilité et d'harmonie évoquée en introduction n'est qu'une illusion friable, une autre façon d'habiter l'existence apparaît en conclusion : faire sa demeure dans le germe de mort qui est inclus dans la vie, accompagner le glissement vers le néant. Le cœur alors s'installe dans le silence, succédané de la paix, et se voue à la mort dont il accepte la sublime souveraineté. L'extase irradiant au début du poème est retrouvée dans une nouvelle unité: demeurant dans la mort, ce cœur est lui-même habité par la mort qui le comble en l'investissant d'une ivresse funèbre (vers 26-28).

Cependant l'ivresse même de ce retour le dénonce comme l'effet d'une complaisance morbide qui ne ménage à son tour qu'un répit illusoire; l'écriture poétique s'abandonne ainsi volontiers aux délices équivoques de la décadence. Mais écrire pour Trakl, c'est, avec une urgence croissante, être en chemin vers une demeure perdue qu'il sait avoir habitée dans sa première enfance. L'éveil survient alors dans les larmes de la rosée après l'ivresse du soir ( $\mathrm{Zu}$ Abend mein Herz). Dans la logique de cet itinéraire poétique, le noble geste du déclin accepté n'est qu'une étape qui se révèle inhabitable. Il faut s'enfoncer plus avant dans la "destruction de novembre ", pourchasser l'être jusqu'au plus profond de sa négation.

16 Avec Herbstliche Heimkehr c'est une nouvelle approche qui est tentée, selon une démarche tâtonnante dont les versions successives de ce texte ont gardé la trace. Le point de départ est la partie centrale du poème précédent, la confrontation avec le souvenir d'une période antérieure de pureté, l'écoute concentrée de l'appel de la demeure perdue. Le décor du jour déclinant qui accompagnait la descente aux ombres intérieures a disparu; l'automne évoqué en titre n'est plus qu'un signifiant métaphorique de la mélancolie, tandis que la décomposition du jardin, qui conserve " un obscur reflet des années enfantines ", est l'image d'une existence qui se défait. Le chemin du retour s'enfonce dans un automne qui n'est plus le décor apaisant offert par une nature complaisante aux deuils poétiques.

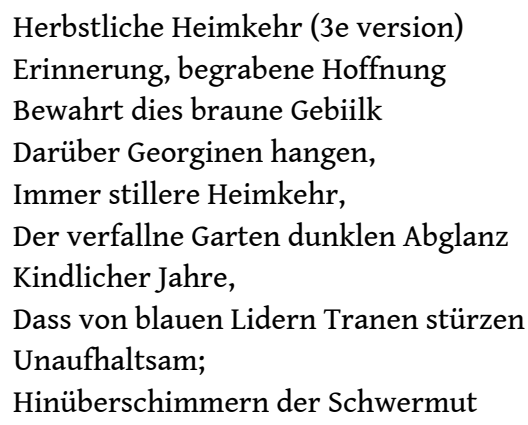


Kristallne Minuten

Zur Nacht ${ }^{7}$. morne pélerinage, à un « retour toujours plus silencieux » au cimetière des espérances mortes (vers 4). Les fleurs, qui étaient le reste d'une vision colorée du monde, prennent l'aspect désolé de lambeaux de souvenir accrochés aux poutres brunies. Avec ses dimensions réduites ce poème présente aussi une économie de moyens, une ascèse stylistique qui a dépouillé les artifices d'un exorcisme esthétique devenu sans effet. Le resserrement autour de l'essentiel s'accompagne de l'abandon de la perspective temporelle. L'allusion au passage sous l'empire du mal a été écartée, de même que l'avènement d'un nouvel «âge d'or » intérieur. C'est sur le présent que cette fois les larmes sont versées («stürzen»), en un flot continu qui semble ne pas s'être tari depuis qu'elles ont jailli pour la première fois (vers 7-8). Le poème se replie sur la pérennité de l'exil et s'enferme dans le jardin dévasté sur lequel la nuit est tombée.

the lueur subsiste de l'apothéose dorée de Heimkehr: elle parvient jusqu'à ce nouveau poème par le détour du texte de An auquel est empruntée l'image finale. Le parcours indirect des mots correspond à celui de la chose, à ce reflet lointain. La lumière qui parvient dans la nuit du présent par le dernier lien de la mélancolie se situe en effet dans un au-delà «hinüberschimmern») défendu par le "seuil pétrifié de douleur »; reflet d'un ailleurs inaccessible, elle manifeste qu'il n'est pas de retour possible - à moins de s'enfoncer précisément dans la souffrance de la mélancolie sur la trace évanescente des "minutes cristallines" de la grâce entrevue (vers 9-11). Là s'ouvre un chemin probable qui, délaissant l'ivresse trompeuse du déclin, explore le désespoir comme dernier témoignage de la dépossession. Ainsi s'amorce la démarche destructrice qui cherche l'objet dans son absence, l'espoir dans la désespérance, le retour dans l'évidence du non-retour.

C'est cet ultime pari que tente Die Heimkehr, à la pointe de l'autre rameau issu de la désagrégation du texte originel. Le poème témoigne d'une mutation du style de l'auteur vers la fin de sa brève carrière: ce qui a été désigné comme le "ton tyrolien" transforme les lentes litanies d'images de la parataxe en déferlements orageux où de brèves lueurs d'espoir s'allument dans les ténèbres: convulsions d'une écriture qui bientôt se taira. Le décor naturel ou plutôt le champ de références de cette poésie qui ne mentionne le monde extérieur que pour dire l'intérieur, est effectivement celui de la haute montagne, peut-être inspiré par un séjour de Trakl au château de Hohenburg que la famille Ficker possédait à Igls près d'Innsbruck. La douce colline où le personnage s'embarquait pour un voyage au pays de l'enfance (Heimkehr) s'est muée en une pente abrupte surplombant une sombre vallée. À l'image de ce paysage alpestre le poème tout entier s'est fait plus âpre. Les poutres fleuries, le petit jardin, vestiges d'un passé enfoui où s'accroche le souvenir, ont disparu et avec eux tout reste de rêve nostalgique. Sur ces cimes d'un combat existentiel il n'est plus de demeure possible, même dans les alanguissements d'un déclin à présent aspiré par le gouffre.

Die Heimkehr

Die Kühle dunkler Jahre,

Schmerz und Hoffnung

Bewahrt zyklopisch Gestein,

Menschenleeres Gebirge,

Des Herbstes goldner Odem,

Abendwolke -

Reinheit! 


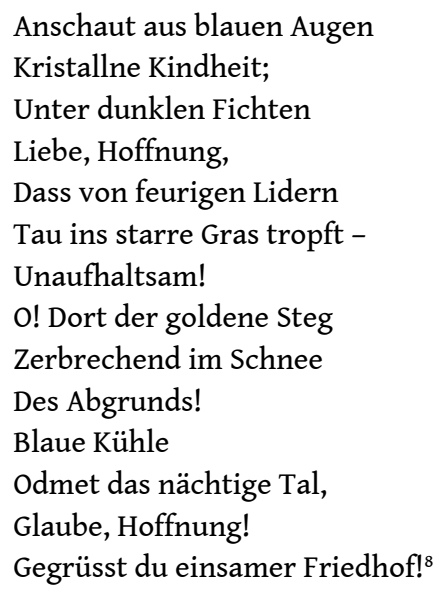

La structure générale des poèmes consacrés au retour subsiste encore dans ce texte sous forme de fragments épars dans ce paysage tourmenté. On en reconnaît les jalons familiers : la persistance d'un souvenir obsédant, l'évocation douloureuse d'une pureté perdue de l'enfance et l'espoir finalement exprimé de l'apaisement trouvé dans une nouvelle demeure. Mais à l'intérieur de ce parcours conservé quelle tragique dégradation! La triple invocation de l'espérance signale paradoxalement les stations d'un désespoir que le temps accentue: dans la réminiscence des sombres années passées l'espérance subsiste aux côtés de la douleur qui la fortifie (strophe 1), dans la vision présente de l'innocence perdue elle n'existe plus, associée à l'amour, que comme le sentiment d'une perte irrémédiable (strophe 2), et pour l'avenir imminent elle n'est plus, soutenue par la foi, qu'un mot jeté au hasard dans le vide (strophe 3).

Ponctuée par ces appels faiblissants à l'espérance cette ultime chronique apporte au chemin du retour des modifications conduisant inéluctablement au nouveau terme tragique du voyage. L'amoncellement cyclopéen de rochers qui prend la place de la maison, le massif désert se dressant là où se trouvait le jardin, prêtent au souvenir la masse d'une stratification géologique scellant de son poids écrasant le caveau des sombres années, cellule d'un prisonnier partagé entre la douleur et l'espoir. La maison familiale, cénotaphe des espérances mortes, a pris les dimensions d'un mausolée formidable qui interdit tout retour (vers 1-4). Pourtant dans ce paysage titanesque réapparaît le couchant automnal avec ses ors, irréel en ces lieux, mais reflet symbolique d'une pureté originelle dont l'appel traverse miraculeusement les strates d'une mémoire pétrifiée (vers 5-7) : et à nouveau s'ouvre le regard bleu de l'enfant qui interroge l'adulte qu'il est devenu (vers 8-9). Pour l'être dépossédé, errant sous les pins sombres, ce regard est un rayon d'amour et d'espoir refusés qui brûle ses paupières en feu (vers 10-12). La rosée que Heimkehr faisait encore pleuvoir des cimes désigne ici des larmes qui ne jaillissent plus des paupières bleues de l'innocence pleurée (Herbstliche Heimkehr), mais s'égouttent inlassablement; l'émotion nostalgique a fait place à une tristesse sans fin, symptôme clinique de la mélancolie profonde, cette gangrène de l'âme : le froid gagne, qui déjà fige l'herbe recevant les pleurs (vers 13-15).

La dernière strophe apporte le cri de la foi qui jaillit au fond du désespoir mené à son terme: elle voit s'écrouler la passerelle d'or qui, comme dans un conte féérique, aurait pu conduire au pays de l'enfance (vers 15-17). L'image «tyrolienne » surimposée du pont de neige s'effondrant dans l'abîme introduit une rupture brutale là où les poèmes précédents proposaient la pente du spleen ou l'ivresse du déclinement. Les lentes décompositions des automnes intérieurs se sont révélées inhabitables : la demeure doit 
être cherchée hors du temps et de ses dégradations organiques ou de ses corruptions morales et psychiques. La vallée nocturne qui s'ouvre et d'où monte un souffle froid indique symboliquement le passage à franchir (vers 18-19). Le bleu encore signale un dernier espoir insensé, dérisoire, comme peut être interprétée en dérision (mais n'estce pas le cas pour tout acte de foi ?) l'invocation de l'espérance dans cette chute vers une « dernière demeure» qui n'a jamais si bien mérité sa dénomination: «Friedhof». C'est en effet le cimetière qui accueille en dernier recours l'errant et lui offre sa paix: le seuil de douleur est franchi, c'est le retour enfin accompli au bout du non-retour.

\section{NOTES}

1. Gaston Bachelard, La poétique de l'espace, P.U.F., Paris, 1957, p. 26.

2. H.K.A., I, p. 102.

3. Cf. le schéma de filiation : H.K.A., II, p. 432.

4. Brenner-Jahrbuch, 1915, p. 10.

5. H.K.A., I, p. 413.

6. H.K.A., I, p. 342.

7. H.K.A., I, p. 349.

8. H.K.A., I, p. 162.

\section{RÉSUMÉS}

De mai à juin 1914 Georg Trakl entreprend, sur les voies du souvenir, un « retour » vers l'enfance, demeure perdue d'une pureté originelle de l'être; cette remémoration que jalonnent trois poèmes (Heimkehr, Herbslliche Heimkehr, Die Heimkehr) constitue une des figures de l'expérience caractéristique du déclin. Le repère utopique de l'absolu retour (Ein Winterabend) donne la mesure du délabrement progressif de l'espoir en une grâce qui se refuse. Ce phénomène de dégradation, souvent signalé dans l'écriture de Trakl, se manifeste ici dans la corruption accélérée d'un groupe de textes organiquement apparentés. D'un poème à l'autre l'ombre gagne avec l'évidence d'un irrémédiable déclin dont l'exploration mnémonique relève la trace. Ainsi se dessine un itinéraire destructeur - métaphore de l'œuvre entière - qui poursuit l'objet dans son absence, l'espoir dans la désespérance, l'être jusqu'au plus profond de sa négation. Abordé sur les pentes d'une complaisante mélancolie, le chemin du retour débouche sur le gouffre d'où monte la vision d'une "dernière demeure » signalant le néant comme ultime refuge de l'être: c'est le retour enfin accompli au bout du non-retour.

Mai-Juni 1914: drei Gedichte G. Trakls zeugen von einer «Heimkehr», die auf dem Weg der Erinnerung in Richtung der Kindheit führt - auf der Suche nach einer verlorenen ursprünglichen Reinheit. Die Trilogie (Heimkehr, Herbstliche Heimkehr, Die Heimkehr) fügt sich somit in den Rahmen 
des für Trakl charakteristischen Untergangsmotivs ein. Am utopischen Entwurf der absoluten Heimkehr (Ein Winterabend) kann der progressive Hoffnungsschwund ermessen werden, der sich in dieser Textreihe kundgibt. Das Phänomen der Zersetzung, das öfters in Verbindung mit Werk und Schreibart des Autors erwähnt wird, läßt sich in verkleinertem Maßstab an dieser Gruppe organisch zusammengewachsener Gedichte aufzeigen. Mit der Aufdeckung eines unverrückbaren Untergangs verdunkelt sich das Bild von einem Text zurn anderen. So zeichnet sich - als Metapher für das Gesamtwerk - ein Weg ab, der die Hoffnung in der Hoffnungslosigkeit, das Wesen bis in seine letzte Negierung sucht. Dieser Weg, der zunächst mit nur leichter Melancholie angetreten wurde, mündet jäh in den Abgrund mit der Vision des «Fried»-hofes als letzter Behausung des Seins, als endgültiger Heimkehr.

\section{AUTEUR}

\section{JEAN-DANIEL KREBS}

Université de Lille IIIfr 